\title{
Overexpression of TMPRSS4 in non-small cell lung cancer is associated with poor prognosis in patients with squamous histology
}

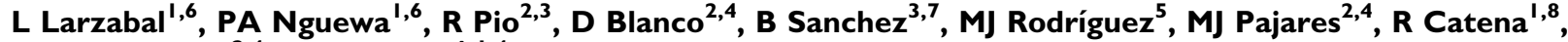 LM Montuenga ${ }^{2,4}$ and A Calvo*,1,4}

'Laboratory of Novel Therapeutic Targets, Division of Oncology, Center for Applied Medical Research, University of Navarra, Pamplona, Spain; ${ }^{2}$ Laboratory of Biomarkers, Division of Oncology, Center for Applied Medical Research, University of Navarra, Pamplona, Spain; ${ }^{3}$ Department of Biochemistry, University of Navarra, Pamplona, Spain; ${ }^{4}$ Department of Histology and Pathology, University of Navarra, Pamplona, Spain;

${ }^{5}$ Research Department, Ingenasa, Madrid, Spain

BACKGROUND: Mortality rates in lung cancer patients have not decreased significantly in recent years, even with the implementation of new therapeutic regimens. One of the main problems is that a large proportion of patients present local or distant metastasis at the time of diagnosis. The need for identification of novel biomarkers and therapeutic targets for a more effective management of lung cancer led us to investigate TMPRSS4, a protease reported to promote tumour growth and metastasis.

MATERIAL AND METHODS: In all, 34 lung cancer cell lines were used to evaluate the TMPRSS4 expression. Cell migration and clonogenic assays, and an in-vivo lung metastasis model were used for functional analysis of the TMPRSS4 downregulation in $\mathrm{H358}, \mathrm{H} 44 \mathrm{I}$ and H2170 cell lines. The TMPRSS4 expression analysis in normal and malignant lung tissue samples was performed by qPCR. Five different microarray-based publicly available expression databases were used to validate our results and to study prognosis. RESULTS: The TMPRSS4 knock down in $\mathrm{H358}, \mathrm{H} 44 \mathrm{I}$ and $\mathrm{H} 2 \mathrm{I} 70$ cells resulted in a significant reduction in proliferation, clonogenic capacity and invasion. A significant $(P<0.05)$ decrease in the lung colonisation and growth was found when mice were injected with TMPRSS4-depleated H358-derived clones, as compared with controls. Expression of TMPRSS4 showed a >30-fold increase $(P<0.00 \mathrm{I})$ in tumours in comparison with non-malignant samples. Levels in tumours with squamous cell carcinoma (SCC) histology were found to be significantly higher $(P<0.00 \mathrm{I})$ than those with adenocarcinoma $(A C)$ histology, which was confirmed in data retrieved from the microarrays. Kaplan-Meier curves demonstrated that high levels of TMPRSS4 were significantly associated $(P=0.017)$ with reduced overall survival in the patients with SCC histology, whereas no correlation was found for the AC histology. CONCLUSION: Our results demonstrate that TMPRSS4 has a role in the lung cancer development. The potential use of TMPRSS4 as a biomarker for lung cancer detection or as a predictor of patient's outcome warrants further investigation.

British Journal of Cancer (201 I) 105, 1608-1614. doi:10.1038/bjc.201 I.432 www.bjcancer.com

(c) 20II Cancer Research UK

Keywords: lung cancer; TMPRSS4; squamous cell carcinoma; serine protease

Lung cancer is a critical problem in public health. It represents the most frequent tumour type in men and the second in women, and the 5-year survival rate remains inferior to $20 \%$ (Jemal et al, 2010). Most of the lung cancer cases belong to the non-small-cell lung cancer (NSCLC) type (85\% of them). Histological subtypes include adenocarcinoma (AC) and squamous cell carcinoma (SCC) accounting for 30 and $50 \%$ of NSCLC, respectively (Youlden et al, 2008). The ACs are malignant epithelial tumours with glandular differentiation, positive for the TTF1 marker. The SCCs

\footnotetext{
*Correspondence: Dr A Calvo; E-mail: acalvo@unav.es

${ }^{6}$ These authors contributed equally to this work

${ }^{7}$ Current address: Roche Diagnostics, S.L. Applied Science, Madrid, Spain

${ }^{8}$ Current address: Department of Cardiothoracic Surgery, Weill Cornell Medical College, Cornell University, New York, New York

Received 14 June 2011; revised 15 September 2011; accepted 19 September 2011
}

are derived from bronchial epithelial cells and express keratins (CK5 and CK6) but not TTF1 (Langer et al, 2010). More than $60 \%$ NSCLC patients present locally advanced, unresectable or metastatic (stage III/IV) tumours at the time of diagnosis, which fatally concludes in death within few months after the diagnosis. Therefore, there is a need for identifying new potential therapeutic targets against which more effective treatments may be developed.

There is a growing evidence demonstrating the critical implication of several genes in cancer development and metastasis, that have been classified as the tumour initiation genes (such as EGFR, KRAS and MYC), metastasis initiation (TWIST, SNAIL and SLUG), progression (metalloproteinases, LOX and ANGPTL4) and metastasis virulence genes (IL6, IL11 and TNF $\alpha$ ) (Nguyen et al, 2009). Deregulation of proteases is considered to be a hallmark of cancer development, because malignant cells require a range of proteolytic activities to enable growth, survival, motility, invasion and digestion of the extracellular matrix (Roy et al, 2009). Different 
studies have indicated that matrix metalloproteinases (MMPs) and serine proteases have an important role in cancer invasion and metastasis (Duffy, 1996). It is well established that proteases contribute to the degradation of the basement membrane and extracellular matrix (Roy et al, 2009). This property allows the tumour cells to invade the surrounding tissue and nearby blood vessels, an essential step in the metastasis development (Gupta and Massague, 2006). Furthermore, MMPs can modulate cell adhesion and proliferation by allowing the bioavailability of growth factors or cell-surface receptors (Fowlkes et al, 1994). In spite of these demonstrated roles in preclinical studies, agents targeting MMPs exhibited poor performances in clinical trials (Chu et al, 2007; Gialeli et al, 2011).

Most members of the serine protease family are either secreted or sequestered in the cytoplasmic organelles. Recently, type II transmembrane serine proteases (TTSPs) that are directly anchored to the plasma membranes have been described (NetzelArnett et al, 2003). These proteases participate in the regulation of cellular signalling events at the plasma membrane and in the extracellular matrix (Hooper et al, 2001). Many of the TTSPs show restricted tissue distribution in normal cells, but their expression is increased during the tumour growth and progression (Benaud et al, 2002).

TMPRSS4 is one of the TTSPs that is upregulated in pancreatic cancer and has been suggested as a diagnostic marker for the malignant thyroid neoplasms (Kebebew et al, 2005). It was demonstrated that TMPRSS4 is implicated in cell invasion, migration and adhesion of cancer cells. Moreover, the overexpression of TMPRSS4 in colon cancer cells was shown to promote epithelial-mesenchymal transition (EMT) through the upregulation of integrin $\alpha 5$, thereby enhancing motility and invasiveness (Jung et al, 2008; Kim et al, 2010). Although preliminary studies using reverse transcription-PCR (RT-PCR) have described the upregulation of this serine protease in few lung cancer specimens compared with the normal tissues (Jung et al, 2008), its expression levels in relation to clinical outcome and its function in lung cancer development is still unclear. In the present work, the expression and biological role of TMPRSS4 were studied in the two principal subtypes of NSCLC. We demonstrate that this protease is implicated in the NSCLC proliferation and migration. Furthermore, we show that TMPRSS4 is highly expressed in tumours compared with the normal lungs, with a higher expression in SCC than in AC. Importantly, we also demonstrate that high TMPRSS4 mRNA levels in SCC are associated with poor prognosis.

\section{MATERIAL AND METHODS}

\section{Patients}

Data from 1022 NSCLC patients were analysed in this study, which belonged to six different cohorts: A $(n=30 ; 11 \mathrm{AC}+18 \mathrm{SCC}+1$ sarcomatoid epidermoid carcinoma histology), B $(n=111 ; 58$ $\mathrm{AC}+53 \mathrm{SCC}), \mathrm{C}(n=171 ; 125 \mathrm{AC}+46 \mathrm{SCC}), \mathrm{D} \quad(n=138 ; 62$ $\mathrm{AC}+76$ SCC $), \mathrm{E}(n=130$; all SCC $)$ and F ( $n=442$; all AC). Cohorts other than A corresponded to series of patients analysed by microarray techniques, which were previously published: B (Bild et al, 2006), C (Potti et al, 2006), D (Lee et al, 2008), E (Raponi et al, 2006) and F (Shedden et al, 2008).

Tumour samples in the group A were obtained from the NSCLC patients who underwent tumour resectional surgery at the Clinica Universidad de Navarra (Pamplona, Spain) and at the Hospital Marqués de Valdecilla (Santander, Spain). In all, 15 tumour samples from this set were obtained with their corresponding matched non-malignant lung tissues; part of the tumour was fixed in $10 \%$ buffered formalin and processed for paraffin embedding and the other portion was frozen for molecular analysis. A $4-\mu \mathrm{m}$ thick section from the paraffin-embedded tissues was cut and analysed by histology after hematoxylin and eosin (H\&E) staining. Sections of frozen samples were cut in a cryostat and analysed histopathologically to ensure that more than $70 \%$ of the tumour samples contained malignant tissue before being used for RNA extraction. Clinical and pathological characteristics of this cohort are shown in Table 1. The study protocol was approved by the Ethical Committee of our institutions.

\section{Cell culture}

All human lung cancer cell lines used in this study were obtained from the the American Type Culture Collection (Manassas, VA, USA) and were maintained in RPMI 1640 with $10 \%$ foetal bovine serum at $37^{\circ} \mathrm{C}$ and $5 \% \mathrm{CO}_{2}$.

$\mathrm{H} 358$ cells were transduced with the $\mathrm{pSFG}_{\mathrm{NES}} \mathrm{TGL}$ retroviral vector as previously described (Ponomarev et al, 2004). Vector containing GFP + cells were selected by fluorescent-activated cell sorting. More than $90 \%$ of the cells were confirmed to express the reporter construct.

\section{Transfection with short-hairpin RNA}

H358, H441 and H2170 cells were transfected with scrambled short-hairpin RNA (shCtrl) or shRNA against TMPRSS4 (shTMP4), kindly donated by Dr Anna Ruiz (Biomedical Research Unit, Vall d'Hebron, Barcelona, Spain). Lipofectamine 2000 (Invitrogen, Carlsbad, CA, USA) was used to perform transfections according to the manufacturer's instructions. After $24 \mathrm{~h}$, cells were treated with $1 \mathrm{mg} \mathrm{ml}^{-1}$ hygromycin (Invitrogen) for 15 days to select the vector-containing cells. After analysis of TMPRSS4 mRNA levels by RT-PCR, clones of H358 (shTMP4-2 and shTMP4-3) and H2170 cell line (shTMP4-8) and H441 cell pools (shTMP4) were isolated, expanded and subjected to proliferation, clonogenic and migration assays.

Table I Clinical and pathological features of patients

\begin{tabular}{|c|c|c|c|c|c|}
\hline Patients & Sex & Age (years) & PTNM & Stage & Histology \\
\hline I & $\mathrm{F}$ & 74 & $\mathrm{~T} 2 \mathrm{NI}$ & $\| B$ & Adenocarcinoma \\
\hline 2 & $\mathrm{~F}$ & 54 & TINO & $\mid A$ & Adenocarcinoma \\
\hline 3 & $M$ & 70 & T2NO & $\mathrm{IB}$ & Adenocarcinoma \\
\hline 4 & $M$ & 77 & T2NO & $\mathrm{IB}$ & Adenocarcinoma \\
\hline 5 & $M$ & 63 & T2NO & $\mathrm{IB}$ & Adenocarcinoma \\
\hline 6 & $M$ & 71 & $\mathrm{~T} 2 \mathrm{~N} 2$ & IV & Adenocarcinoma \\
\hline 7 & $M$ & 56 & $\mathrm{~T} 2 \mathrm{~N} 2$ & $\| \mathrm{A}$ & Adenocarcinoma \\
\hline 8 & $M$ & 60 & T2No & IB & Adenocarcinoma \\
\hline 9 & $M$ & 77 & $\mathrm{~T} 2 \mathrm{~N} 2$ & $\| A$ & Adenocarcinoma \\
\hline 10 & $M$ & 54 & T2NO & $\mathrm{IB}$ & Adenocarcinoma \\
\hline|| & $\mathrm{F}$ & 62 & T2NO & $\mathrm{IB}$ & Adenocarcinoma \\
\hline 12 & $M$ & 78 & TINO & $\mid A$ & Squamous carcinoma \\
\hline 13 & $M$ & 49 & TINO & $\mid A$ & Squamous carcinoma \\
\hline 14 & M & 66 & T2NO & $\mathrm{IB}$ & Squamous carcinoma \\
\hline 15 & $M$ & 59 & TINO & $\mid A$ & Squamous carcinoma \\
\hline 16 & $M$ & 79 & TINO & $\mid A$ & Squamous carcinoma \\
\hline 17 & $M$ & 63 & $\mathrm{~T} 2 \mathrm{NI}$ & $\| B$ & Squamous carcinoma \\
\hline 18 & $M$ & 76 & $\mathrm{~T} 2 \mathrm{NI}$ & $\| B$ & Squamous carcinoma \\
\hline 19 & $M$ & 77 & $\mathrm{~T} 2 \mathrm{NI}$ & $\| B$ & Squamous carcinoma \\
\hline 20 & $M$ & 81 & T2NO & $\mathrm{IB}$ & Squamous carcinoma \\
\hline 21 & $M$ & 63 & $\mathrm{~T} 2 \mathrm{NO}$ & $\mid \mathrm{B}$ & Squamous carcinoma \\
\hline 22 & $M$ & 76 & T2NO & $\mathrm{IB}$ & Squamous carcinoma \\
\hline 23 & $M$ & 78 & $\mathrm{~T} 2 \mathrm{NI}$ & $\| B$ & Squamous carcinoma \\
\hline 24 & M & 75 & T3N2 & $\| A$ & Squamous carcinoma \\
\hline 25 & M & 76 & $\mathrm{~T} 2 \mathrm{~N} 2$ & $\| \mathrm{A}$ & $\begin{array}{l}\text { Sarcomatoid epidermoid } \\
\text { carcinoma }\end{array}$ \\
\hline 26 & $M$ & 75 & $\mathrm{~T} 3 \mathrm{NI}$ & $\| A$ & Squamous carcinoma \\
\hline 27 & $\mathrm{~F}$ & 66 & T2NO & $\mathrm{IB}$ & Squamous carcinoma \\
\hline 28 & $M$ & 87 & T2NO & $\mathrm{IB}$ & Squamous carcinoma \\
\hline 29 & $M$ & 69 & $\mathrm{~T} 2 \mathrm{~N} 2$ & $\| A$ & Squamous carcinoma \\
\hline 30 & $M$ & 49 & $\mathrm{~T} 1 \mathrm{~N} 2$ & $\| A$ & Squamous carcinoma \\
\hline
\end{tabular}




\section{RT - PCR}

Total RNA was isolated using the RNEasy Minikit (Qiagen, Madrid, Spain). After DNase I treatment, reverse transcription was performed with Superscript II reverse transcriptase (Invitrogen) to generate complementary DNA. The qRT - PCR was performed with an Applied Biosystems 7900 Real-time PCR System. In human lung specimens, the measurement of TMPRSS4 expression was carried out with the TaqMan Gene Expression Assays (Applied Biosystems, Foster City, CA, USA), according to the manufacturer's protocol. This plate contains the following human endogenous genes: $\beta$-actin, HPRT and IPO8. To normalise gene expression in the tissues samples, IPO8 was selected as an accurate control gene, as previously described (Nguewa et al, 2008). In the cell lines, RT - PCR reactions were carried out with SYBR Green PCR Master Mix (Applied Biosystems) and GAPDH levels were used as controls. The mean cycle threshold value $(\mathrm{Ct})$ for the gene of interest normalised to the $\mathrm{Ct}$ value of the housekeeping gene was used to calculate gene expression values.

\section{Proliferation and clonogenic assays}

To determine proliferation of $\mathrm{H} 358, \mathrm{H} 441$ and $\mathrm{H} 2170$ cells and their shRNA clones, 1200 cells for H358 and H441, and 2000 cells for $\mathrm{H} 2170$ per well were seeded in 96-well plates. After 96 hours of plating, MTT assays were performed (Roche, Palo Alto, CA, USA) following the manufacturer's protocol. To evaluate the clonogenic potential of $\mathrm{H} 358$ and $\mathrm{H} 441$ cells and their respective shRNA clones, 300 cells per well were plated into six-well plates. After 15 days of culture, colonies were fixed with $10 \%$ buffered formalin and stained with $2 \%$ crystal violet. The number of colonies was determined and data are presented as percentage with respect to parental cell line.

\section{Wound healing in-vitro migration assay}

Cell migration was evaluated with an in-vitro model of wound healing. Cells were grown until confluence and monolayers were scraped with a 20 -p micropipette tip. After $24 \mathrm{~h}$, pictures of the wounds were taken with a Nikon Eclipse photomicroscope (Nikon, Kingston, UK) using the ACT-2U1.6 software (Nikon, Kingston, $\mathrm{UK})$. The distance between the wound edges was measured with the Image J analysis software (NIH Image, Bethesda, USA). Six wells per condition were used.

\section{Animal model and histology}

To determine the role of TMPRSS4 in lung metastasis, NOD SCID ILR $\gamma 2$ (NSG) mice were used. All procedures were carried out in accordance with the guidelines for animal experimentation of our Institution (University of Navarra), under approved protocols.

In all, $5 \times 10^{5}$ of H358 shCtrl, shTMP4- 2 or shTMP4-3 cell clones were injected into the tail vein. To monitor lung metastasis by bioluminescence, mice were anaesthetised with a mixture of ketamine $\left(150 \mathrm{mg} \mathrm{kg}^{-1}\right)$ and xylazine $\left(10 \mathrm{mg} \mathrm{kg}^{-1}\right)$ intraperitoneally. Then, $1.5 \mathrm{mg}$ D-luciferin in $100 \mu \mathrm{l}$ of PBS was injected. Imaging was completed at $2 \mathrm{~min}$ for each group of mice with a Xenogen IVIS system coupled to Living Image acquisition and analysis software (Xenogen Inc., Alameda, CA, USA). Photon flux was calculated for each mouse by using a circular region of interest for each lung. The background value (from luciferin-injected mice with no tumour cells) was subtracted from each measurement.

The lung tissues were fixed in $10 \%$ buffered formalin, embedded in paraffin and sectioned ( $5 \mu \mathrm{m}$ in thickness). Slides were stained with $\mathrm{H} \& \mathrm{E}$, and histological images were captured with a Leica microscope (Wetzlar, Germany).

\section{Microarray data analysis and statistics}

Data from the lung cancer microarrays previously described (Bild et al, 2006; Potti et al, 2006; Raponi et al, 2006; Lee et al, 2008;
Shedden et al, 2008) were analysed. The statistical analyses were performed with GraphPad Prism 5.0 (GraphPad Software Inc., San Diego, CA, USA) and R 2.6.0 sofware (www.R-project.org/). Significant differences in TMPRSS4 gene expression between lung adenocarcinomas and squamous carcinomas or between the tumour and non-malignant specimens were analysed by unpaired two-tailed Student's $t$-test. Kaplan-Meier plots were used to illustrate differences in progression according to the level of TMPRSS4 gene expression. The TMPRSS4 expression was dichotomised using the tertile values (low expression included in tertile 1 and high expression in tertiles 2 and 3). Differences in overall survival (OS) (censored at 48 months) were compared using the log-rank test. $P$-values $<0.05$ were considered statistically significant.

\section{RESULTS}

\section{TMPRSS4 is expressed in lung cancer cell lines}

We first investigated the expression of TMPRSS4 in 34 human lung small cell lung cancer (SCLC) and NSCLC cell lines using RT - PCR. The TMPRSS4 mRNA was detected in half (17 out of 34 ) of the cell lines tested (Figure 1). The TMPRSS4 was highly expressed in 14 cell lines: Eight of them were derived from AC (H441, H2087, CALU3, H1648, HCC827, H358, PC14 and H322), one from SCC (H2170), three from SCLC (H187, H209 and H510), one from large cell carcinoma (97TM1) and one from carcinoid (H727). As TMPRSS4 had been linked to tumour development, we decided to study the role of this protein in lung cancer. To this end, we selected two cell lines derived from AC (H358 and H441) and one derived from SSC (H2170), with evident high expression of TMPRSS4 mRNA.

\section{TMPRSS4 inhibition reduces lung cancer cell proliferation and migration}

To inhibit endogenous high TMPRSS4 levels, these three cell lines (H358, H441 and H2170) were transfected with control shorthairping RNA (shCtrl) or TMPRSS4-specific shRNA (shTMP4). As explained in Material and Methods, in the case of H358 and H2170 cells, some clones (shTMP4-2 and shTMP4-3 for H358 and shTMP4-8 for H2170) with a reduction higher than $60 \%$ in TMPRSS4 levels (Figure 2A) were selected and used for further experiments. For H441 cells, the whole cell population carrying the vector, with more than 70\% reduction in TMPRSS4 expression was used (Figure 2A). This approach avoided a possible selection of particular clones with slow proliferation rates. Next, an MTT assay was carried out to assess the effect of TMPRSS4 knockdown on cell growth. As shown in Figure 2B, a shRNA-mediated depletion of
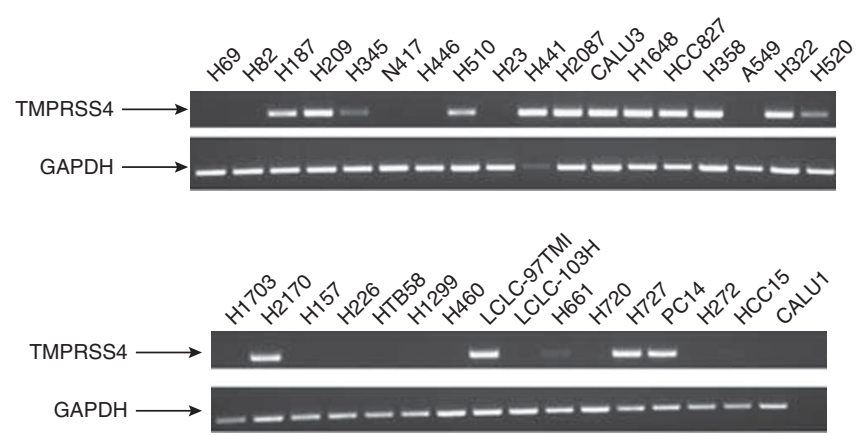

Figure I TMPRSS4 is expressed in lung cancer cell lines. The RT-PCR analysis for TMPRSS4 expression in 34 human lung cancer cell lines. In all, 17 cell lines were positive for this gene. The GAPDH was used as an internal control. 
A

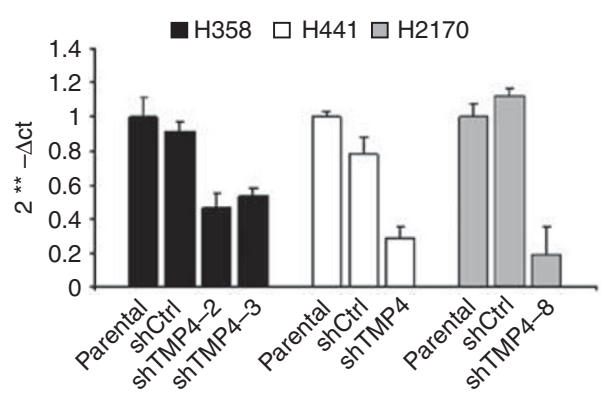

C

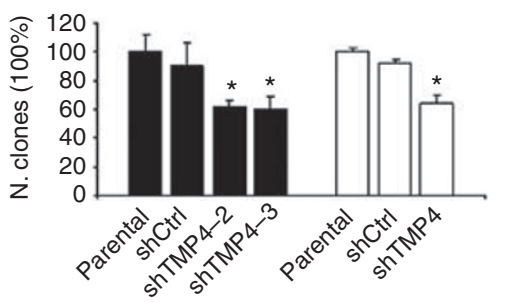

D

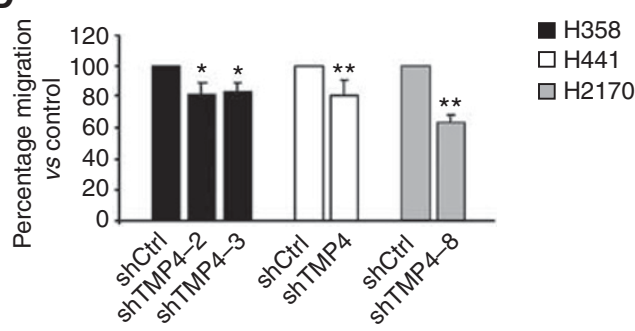

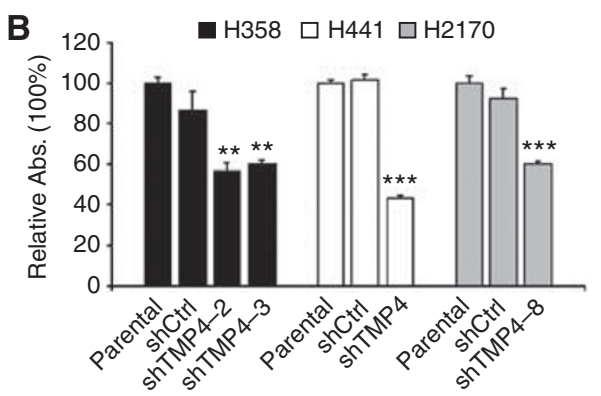
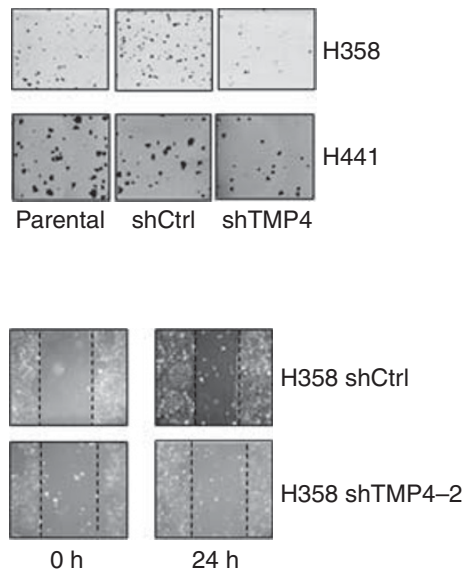

Figure 2 Knockdown of TMPRSS4 by shRNA reduces proliferation, clonogenic potential and migration of lung cancer cell lines. (A) Expression of TMPRSS4 in H358, H44I and $\mathrm{H} 2170$ cell lines after transfection with control shRNA or TMPRSS4-specific shRNA (shTMP4 for simplification), measured by qPCR. The GAPDH was used as internal control. (B) After $96 \mathrm{~h}$ of plating MTT assay was performed. An inhibition in the proliferation of H358, H44I and $\mathrm{H} 2170$ cells transfected with TMPRSS4-specific shRNA $(P<0.01)$ was observed. $(\mathbf{C})$ Clonogenic assay showed a lower number of clones in H358 and $\mathrm{H} 44 \mathrm{I}$ shTMP4 cells compared with the control cells. (D) Migration of $\mathrm{H} 358(P<0.05), \mathrm{H} 44 \mathrm{I}(P<0.0 \mathrm{I})$ and $\mathrm{H} 2 \mathrm{I} 70(P<0.0 \mathrm{I})$ cells was impaired (about $20 \%)$ by the suppression of TMPRSS4. *P $<0.05$; ** $P<0.01$; **** $P<0.001$.

TMPRSS4 resulted in a strong reduction of cell growth in clones from the three cell lines $(P<0.01)$. In the clonogenic assay, we also observed a significant reduction in the number of colonies in H358 shTMP4-2 and shTMP4-3, and in H441-shTMP4 cells compared with the control cells (Figure 2C). Clonogenic experiments could not be performed with the $\mathrm{H} 2170$ cell line and its shRNA clones because, in our hands, these cells did not form colonies with a sufficient size so as to be accurately quantified. These data indicate that TMPRSS4 inhibition decreases cell growth in lung cancer cells.

We next investigated whether TMPRSS4 would modify unidirectional cell migration in the lung cancer cells. For this purpose, parental H358, H441 and H2170 cells or cells carrying the shRNA vectors were subjected to a wound-healing assay. As shown in Figure 2D, $24 \mathrm{~h}$ after plating, downregulation of TMPRSS4 caused a $20 \%$ reduction in the ability of $\mathrm{H} 358, \mathrm{H} 441$ and $\mathrm{H} 2170$ cells to migrate. Therefore, TMPRSS4 participates in the lung cancer cell migration.

\section{Downregulation of TMPRSS4 results in a decrease in lung metastasis}

In-vivo experiments were performed to determine the role of TMPRSS4 in lung cancer development. The NSG mice were injected into the tail vein with $5 \times 10^{5} \mathrm{H} 358$ control cells, shTMP4-2 or shTMP4-3 cell clones. Bioluminescence image analysis was performed to measure tumour growth. The subsequent photon emission quantification indicated a significant reduction $(P<0.05)$ of the tumour growth at day 10 after the cell injection (Figure $3 \mathrm{~A}$ ). At the end of the study (day 35), animals were killed and the lungs were examined for metastatic lesions. Metastatic foci in the lungs of all mice were evaluated by histological analysis (Figure 3B). In mice injected with $\mathrm{H} 358$ shCtrl cells, we observed an average of $20.17 \pm 9.94$ metastatic foci per mice, while in mice injected with H358 shTMP4 cell clones we found $13.33 \pm 5.55$ (shTMP4-2) and $13.86 \pm 14.6$ (shTMP4-3). Although there were no statistical differences, low levels of TMPRSS4 tended to be associated with fewer metastatic foci per mice. Besides, large metastatic areas with highly angiogenic and haemorrhagic areas could be seen in control mice, but not in the shTMP4 group (Figure $3 \mathrm{~B}$ ). These results suggest a role of TMPRSS4 in lung tumour growth and metastasis formation.

\section{TMPRSS4 mRNA levels are dramatically increased in} human lung cancer samples compared with non-malignant tissue, particularly in squamous cell carcinomas

The aforementioned results suggested a protumorigenic role of TMPRSS4 in lung cancer. Because a reliable antibody against TMPRSS4 is not yet available, we evaluated mRNA levels of this protease by $\mathrm{qRT}-\mathrm{PCR}$ in a clinical sample set including normal $(n=15)$ and NSCLC $(n=30 ; 11$ AC +18 SCC +1 sarcomatoid epidermoid carcinoma histology) samples. This cohort of patients included stage I-IV tumours (Table 1). Figure 4A shows that TMPRSS4 expression was dramatically upregulated ( $>30$-fold) in lung tumours compared with normal lungs $(P<0.001)$. In this cohort of patients, those with SCC histology showed significantly 


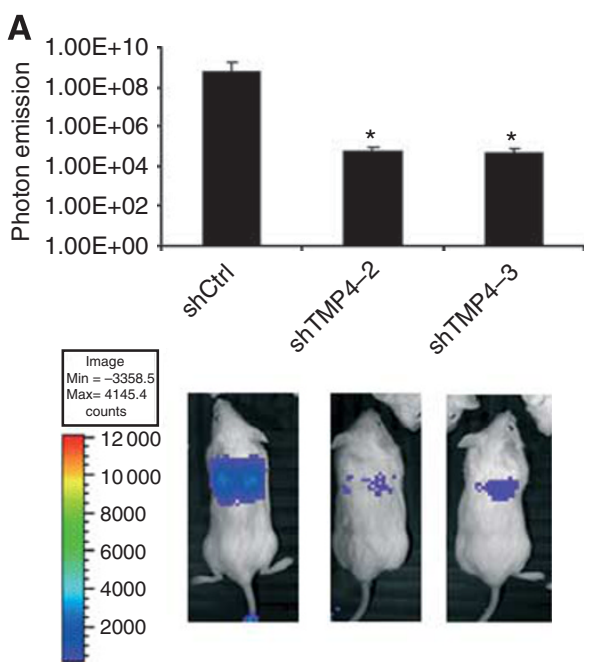

B
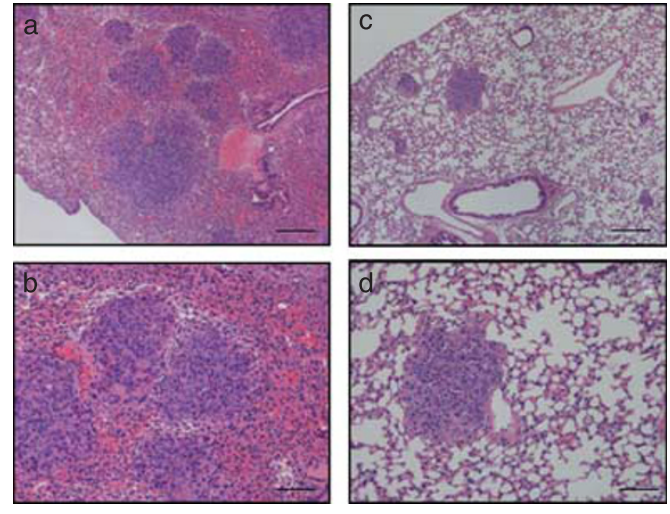

shCtrl

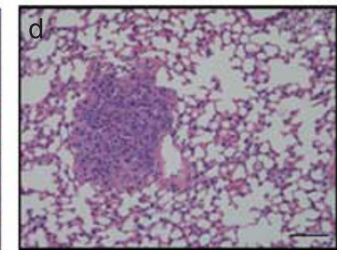

shTMP4-2

Figure 3 Downregulation of TMPRSS4 results in a decrease in lung tumour growth. (A) Representative luminometric images of mice injected with $5 \times 10^{5} \mathrm{H} 358$ control or shTMP4 cell clones in the tail vein. Photon emission quantification revealed a significant reduction in tumour growth in the groups injected with shTMP4-2 and shTMP4-3 cell clones $(P<0.05)$. (B) Representative histological images of lungs from mice injected with H358 shCtrl $(a, b)$ or shTMP4-2 $(c, d)$ cell clones. Large tumours with highly angiogenic and haemorrhagic areas could be seen in control mice, but not in the shTMP4 group. Bars: a, c: $300 \mu \mathrm{m} ; b$, d: $120 \mu \mathrm{m}$. $* P<0.05$.

A
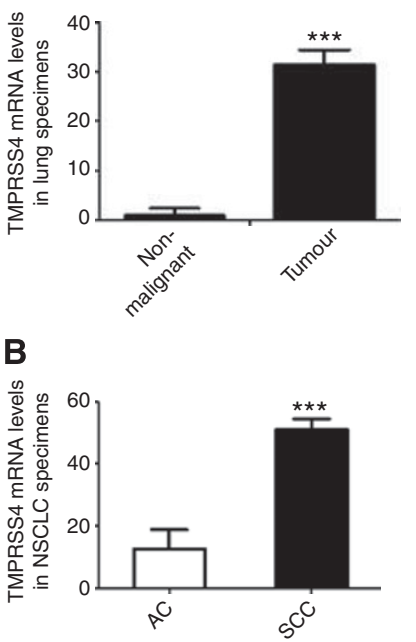

C
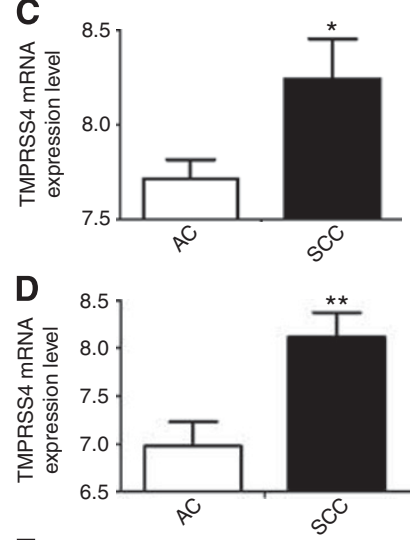

E

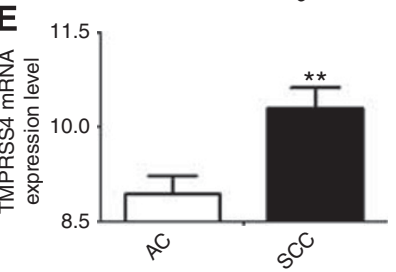

A

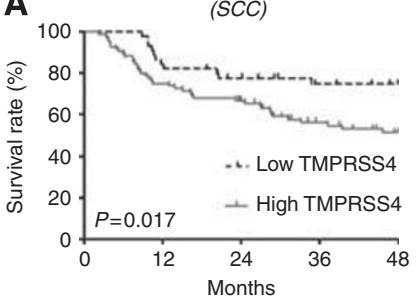

B

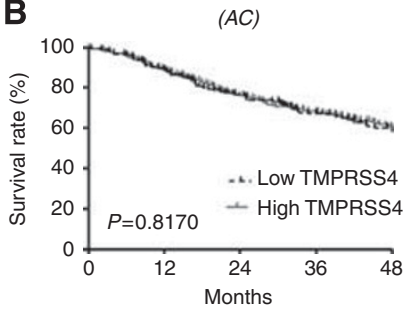

Figure 5 TMPRSS4 levels and clinical outcome. (A) High expression of TMPRSS4 significantly correlated with poor OS in lung SCC patients from Raponi et al, $(P=0.017)$. (B) No association with $O S$ in patients with $A C$ histology was observed from the data published by Shedden et al (2008) $(P=0.817)$.

comparisons between TMPRSS4 expression in both the types of tumour histologies could be performed. Confirming our previous results, lung SCC exhibited significantly higher levels of TMPRSS4 than $\mathrm{AC}$ (Figure 4C-E) in the three databases.

\section{High mRNA levels of TMPRSS4 correlated with poor outcomes}

Figure 4 TMPRSS4 expression in clinical lung specimens. (A) TMPRSS4 levels were significantly higher $(>30$-fold increase, $P<0.00$ I) in tumours than in non-malignant samples, and $(\mathbf{B})$ in SCC than in AC lung tumour specimens $(P<0.00 \mathrm{I})$. $(\mathbf{C}-\mathbf{E})$ To validate these results, an analysis was carried out in three additional cohorts of patients. Again, tumours with SCC histology showed significantly more TMPRSS4 expression than tumours with AC histology, in these three different databases: (C) Potti et al (2006) (D) Lee et $a l$ (2008) and (E) Bild et al (2006). ${ }^{*} P<0.05$; ${ }^{*} * P<0.0$ I; **** $P<0.00$ I.

higher TMPRSS4 levels than those with AC histology $(P<0.001)$ (Figure 4B).

In order to validate these results in larger and independent series of patients, we retrieved TMPRSS4 data from three different microarray expression databases with both AC and SCC cases (Bild et al, 2006; Potti et al, 2006; Lee et al, 2008). These databases included a total number of 420 NSCLC patients. No results from normal lung were available for any of the databases and only

We then investigated whether the expression of TMPRSS4 was associated with clinical outcome in NSCLC, depending on histology. For this purpose, we used databases with a large number of patients that specifically included either AC or SCC, where information on OS was available. Two databases, one for SCC (Raponi et al, 2006) and the other one for AC (Shedden et al, 2008) were used. In SCC, high levels of TMPRSS4 significantly correlated with reduced OS (Figure $5 \mathrm{~A}, P=0.017$ ). In the case of AC, consistent with the lower levels of TMPRSS4 found in patients (Figure 4), there was no discrimination between both of the subgroups (high and low mRNA TMPRSS4 levels) and prognosis $(P=0.8170)$ (Figure 5B). We also analysed mRNA TMPRSS4 levels in relation to disease-free survival (DFS) in the AC series (Shedden et al, 2008), as data in SCC (Raponi et al, 2006) were not available. In agreement with the results found for OS in patients with $\mathrm{AC}$ histology, no statistical relationship was observed. We conclude that high TMPRSS4 mRNA levels are associated with poor prognosis in the NSCLC patients with SCC histology. 


\section{DISCUSSION}

The present study reports the novel finding that TMPRSS4 is highly overexpressed in NSCLC and has a role in lung cancer development. We show here first evidence that TMPRSS4 is highly expressed in lung cancer cell lines and patients, and that such expression is significantly associated with poor prognosis in the case of SCC histology. Recent studies in thyroid, pancreatic and colon carcinoma showed that TMPRSS4 has a protumorigenic and metastatic role in these tumour types (Wallrapp et al, 2000; Kebebew et al, 2005). Because of the critical need for identifying new therapeutic targets for advanced lung cancer, and the fact that a previous study in colon cancer suggested that TMPRSS4 might also be involved in lung carcinogenesis, we hypothesised that this protease would increase the malignant behaviour of NSCLC cells. Analysis of the expression of TMPRSS4 mRNA levels in 34 lung cancer cell lines revealed that the majority of positive cells corresponded to AC histology. Three cell lines, two derived from AC (H358 and H441) and one derived from SSC (H2170) were selected for functional assays, as they showed high TMPRSS4 levels. Interestingly, depletion of TMPRSS4 in these cell lines using shRNA technology significantly affected their proliferation and clonogenic potential. These results indicate that this serine protease induces cell growth in NSCLC.

Metastatic implications of TMPRSS4 have been studied in colon cancer (Jung et al, 2008), which prompted us to analyse cell migration and metastasis in vivo. We have demonstrated that knockdown of TMPRSS4 by shRNA reduces significantly cell migration in vitro in $\mathrm{H} 358, \mathrm{H} 441$ and $\mathrm{H} 2170$ cell lines. Furthermore, in an in vivo model of lung cancer metastasis we show that inhibition of TMPRSS4 in luciferase-expressing H358 cells results in a decline in luminometric signals in the lungs. The TMPRSS4 downregulates epithelial markers, such as E-cadherin and P-cadherin, and upregulates mesenchymal markers that induce EMT in colon cancer cells (Jung et al, 2008). Our findings reinforce the main role of proteases (including TMPRSS4) in invasion and metastasis development.

We further evaluated TMPRSS4 expression in both the lung tumour and non-malignant samples. Tumour specimens exhibited much higher levels than normal lungs. In addition, we found that TMPRSS4 levels were higher in SCC patients as compared with those with AC histology, both in our series of patients as well as in microarray-retrieved data (Bild et al, 2006; Potti et al, 2006; Lee et al, 2008). This is a surprising result that contrasts with the predominant expression of TMPRSS4 in AC-derived cell lines. It is possible that the conditions of the tumour microenvironment switches on TMPRSS4 expression in SCC cells in vivo and that these conditions are not reproduced in in vitro cultures.
Alternatively, stromal cells within the squamous tumour may be responsible for TMPRSS4 expression, instead of tumour cells themselves. Reliable antibodies should be developed in order to ascertain the precise location of TMPRSS4 in SCC tumours.

The next question we addressed was whether the expression of TMPRSS4 was associated with the clinical outcome in NSCLC, especially in SCC, where TMPRSS4 levels were found to be the highest. We hypothesised that an increase in this protease would worsen clinical outcome in the NSCLC patients. For this analysis, we used a database (Raponi et al, 2006) that includes a large and well-characterised series of SCC patients. Results indicate that high TMPRSS4 mRNA levels are significantly associated with poor prognosis for SCC. These results show for the first time (to the best of our knowledge) an association between high TMPRSS4 and poor prognosis.

All these data support our hypothesis that TMPRSS4 has an important role in lung cancer, promoting cell proliferation, tumour growth and invasion. Based on our results in patients, we propose TMPRSS4 as a putative biological marker for NSCLC (particularly for SCC histology), and as an indicator of poor prognosis. The development of specific antibodies (which are lacking at this moment) against this protease will allow further studies by immunohistochemistry in independent and larger series of patients to confirm our mRNA-based results. In addition, TMPRSS4 could also be considered as a therapeutic target. Although therapeutic efficacy of MMPs inhibitors rendered unsatisfactory results in clinical trials (Chu et al, 2007; Gialeli et al, 2011), it may not be the case for the family of serine proteases, including TMPRSS4. Future strategies for the development of TMPRSS4-targeting therapies (that may include blocking peptides or antibodies) should be explored. What seems clear is that the identification of squamous NSCLC-specific proteins (such as TMPRSS4) opens new avenues for diagnosis and treatment with this type of disease.

\section{ACKNOWLEDGEMENTS}

This work has been funded by 'UTE project CIMA', ISCIII-RTICC RD06/0020/0066 grant; Ministerio de Educacion y Ciencia Grant SAF2007-64184 (to AC), Ministerio de Industria (Programa Ingenio 2010, CENIT Ref. Oncnosis), Gobierno de Navarra Salud (2540/2008 to PAN). LL was supported by a Gobierno Vasco fellowship. We would also like to thank Dr Anna Ruiz (Biomedical Research Unit, Vall d'Hebron, Barcelona, Spain) for kindly providing the shRNA constructs. The TaqMan HT work has been supported by the CENIT project.

\section{REFERENCES}

Benaud CM, Oberst M, Dickson RB, Lin CY (2002) Deregulated activation of matriptase in breast cancer cells. Clin Exp Metastasis 19(7): $639-649$

Bild AH, Yao G, Chang JT, Wang Q, Potti A, Chasse D, Joshi MB, Harpole D, Lancaster JM, Berchuck A, Olson Jr JA, Marks JR, Dressman HK, West M, Nevins JR (2006) Oncogenic pathway signatures in human cancers as a guide to targeted therapies. Nature 439(7074): $353-357$

Chu QS, Forouzesh B, Syed S, Mita M, Schwartz G, Cooper J, Curtright J, Rowinsky EK (2007) A phase II and pharmacological study of the matrix metalloproteinase inhibitor (MMPI) COL-3 in patients with advanced soft tissue sarcomas. Invest New Drugs 25(4): 359-367

Duffy MJ (1996) Proteases as prognostic markers in cancer. Clin Cancer Res 2(4): $613-618$

Fowlkes JL, Enghild JJ, Suzuki K, Nagase H (1994) Matrix metalloproteinases degrade insulin-like growth factor-binding protein-3 in dermal fibroblast cultures. J Biol Chem 269(41): 25742-25746
Gialeli C, Theocharis AD, Karamanos NK (2011) Roles of matrix metalloproteinases in cancer progression and their pharmacological targeting. Febs J 278(1): 16-27

Gupta GP, Massague J (2006) Cancer metastasis: building a framework. Cell 127(4): $679-695$

Hooper JD, Clements JA, Quigley JP, Antalis TM (2001) Type II transmembrane serine proteases. insights into an emerging class of cell surface proteolytic enzymes. J Biol Chem 276(2): $857-860$

Jemal A, Siegel R, Xu J, Ward E (2010) Cancer statistics, 2010. CA Cancer J Clin 60(5): $277-300$

Jung H, Lee KP, Park SJ, Park JH, Jang YS, Choi SY, Jung JG, Jo K, Park DY, Yoon JH, Lim DS, Hong GR, Choi C, Park YK, Lee JW, Hong HJ, Kim S, Park YW (2008) TMPRSS4 promotes invasion, migration and metastasis of human tumor cells by facilitating an epithelial-mesenchymal transition. Oncogene 27(18): 2635-2647

Kebebew E, Peng M, Reiff E, Duh QY, Clark OH, McMillan A (2005) ECM1 and TMPRSS4 are diagnostic markers of malignant thyroid neoplasms 
and improve the accuracy of fine needle aspiration biopsy. Ann Surg 242(3): $353-361$; discussion $361-3$

Kim S, Kang HY, Nam EH, Choi MS, Zhao XF, Hong CS, Lee JW, Lee JH, Park YK (2010) TMPRSS4 induces invasion and epithelial-mesenchymal transition through upregulation of integrin alpha5 and its signaling pathways. Carcinogenesis 31(4): $597-606$

Langer CJ, Besse B, Gualberto A, Brambilla E, Soria JC (2010) The evolving role of histology in the management of advanced non-small-cell lung cancer. J Clin Oncol 28(36): 5311-5320

Lee ES, Son DS, Kim SH, Lee J, Jo J, Han J, Kim H, Lee HJ, Choi HY, Jung Y, Park M, Lim YS, Kim K, Shim Y, Kim BC, Lee K, Huh N, Ko C, Park K, Lee JW, Choi YS, Kim J (2008) Prediction of recurrence-free survival in postoperative non-small cell lung cancer patients by using an integrated model of clinical information and gene expression. Clin Cancer Res 14(22): $7397-7404$

Netzel-Arnett S, Hooper JD, Szabo R, Madison EL, Quigley JP, Bugge TH, Antalis TM (2003) Membrane anchored serine proteases: a rapidly expanding group of cell surface proteolytic enzymes with potential roles in cancer. Cancer Metastasis Rev 22(2-3): 237-258

Nguewa PA, Agorreta J, Blanco D, Lozano MD, Gomez-Roman J, Sanchez BA, Valles I, Pajares MJ, Pio R, Rodriguez MJ, Montuenga LM, Calvo A (2008) Identification of importin 8 (IPO8) as the most accurate reference gene for the clinicopathological analysis of lung specimens. BMC Mol Biol 9: 103

Nguyen DX, Bos PD, Massague J (2009) Metastasis: from dissemination to organ-specific colonization. Nat Rev Cancer 9(4): 274-284

Ponomarev V, Doubrovin M, Serganova I, Vider J, Shavrin A, Beresten T, Ivanova A, Ageyeva L, Tourkova V, Balatoni J, Bornmann W, Blasberg R, Gelovani Tjuvajev J (2004) A novel triple-modality reporter gene for whole-body fluorescent, bioluminescent, and nuclear noninvasive imaging. Eur J Nucl Med Mol Imaging 31(5): 740-751

Potti A, Mukherjee S, Petersen R, Dressman HK, Bild A, Koontz J, Kratzke R, Watson MA, Kelley M, Ginsburg GS, West M, Harpole Jr DH., Nevins JR (2006) A genomic strategy to refine prognosis in early-stage non-small-cell lung cancer. $N$ Engl J Med 355(6): 570-580

Raponi M, Zhang Y, Yu J, Chen G, Lee G, Taylor JM, Macdonald J, Thomas D, Moskaluk C, Wang Y, Beer DG (2006) Gene expression signatures for predicting prognosis of squamous cell and adenocarcinomas of the lung. Cancer Res 66(15): 7466-7472

Roy R, Yang J, Moses MA (2009) Matrix metalloproteinases as novel biomarkers and potential therapeutic targets in human cancer. J Clin Oncol 27(31): 5287-5297

Shedden K, Taylor JM, Enkemann SA, Tsao MS, Yeatman TJ, Gerald WL, Eschrich S, Jurisica I, Giordano TJ, Misek DE, Chang AC, Zhu CQ, Strumpf D, Hanash S, Shepherd FA, Ding K, Seymour L, Naoki K, Pennell N, Weir B, Verhaak R, Ladd-Acosta C, Golub T, Gruidl M, Sharma A, Szoke J, Zakowski M, Rusch V, Kris M, Viale A, Motoi N, Travis W, Conley B, Seshan VE, Meyerson M, Kuick R, Dobbin KK, Lively T, Jacobson JW, Beer DG (2008) Gene expression-based survival prediction in lung adenocarcinoma: a multi-site, blinded validation study. Nat Med 14(8): $822-827$

Wallrapp C, Hahnel S, Muller-Pillasch F, Burghardt B, Iwamura T, Ruthenburger M, Lerch MM, Adler G, Gress TM (2000) A novel transmembrane serine protease (TMPRSS3) overexpressed in pancreatic cancer. Cancer Res 60(10): 2602-2606

Youlden DR, Cramb SM, Baade PD (2008) The International Epidemiology of Lung Cancer: geographical distribution and secular trends. J Thorac Oncol 3(8): $819-831$

This work is published under the standard license to publish agreement. After 12 months the work will become freely available and the license terms will switch to a Creative Commons Attribution-NonCommercial-Share Alike 3.0 Unported License. 